\title{
Middle School Students' Patterning Performance on Semi-Free Generalization Tasks
}

\author{
F. D. Rivera and Joanne Rossi Becker \\ Department of Mathematics \& Statistics \\ San Jose State University \\ 1 Washington Square \\ San Jose, CA 95192 USA \\ Ferdinand.rivera@sjsu.edu, (408) 924-5170 \\ joanne.rossibecker@sjsu.edu, (408) 924 - 5112
}

The research that is reported in this document has been funded by the National Science

Foundation under Grant Number DRL 0448649 awarded to the first author. All the views and opinions expressed in this report are solely the authors' responsibility and do not necessarily reflect the views of the foundation. 


\begin{abstract}
This longitudinal study empirically addresses the issue of structure construction and justification among a class of US seventh and eighth-grade Algebra 1 students (mean age of 12.5 years) in the context of novel semi-free pattern generalization (PG) tasks before and after a teaching experiment that emphasized a multiplicative thinking approach to patterns. We compared the students' PG responses before and after the experiment and found that (1) one source of variability in their abduced structural processing was in part due to an initial conceptual preference toward thinking either in parts or in wholes and (2) a multiplicative understanding of structures significantly aided them in PG conversion (e.g., from the visual to the alphanumeric) and processing (e.g., from nonstandard to standard function-based formulas). Our findings provide both necessary and sufficient conditions for constructing, establishing, and justifying valid structures in the case of (semi-) free figural patterning tasks.
\end{abstract}




\section{Introduction}

Children and adults have been documented as having a natural disposition toward pattern generalization (“PG;” Cadez \& Kolar, 2015; Jurdak \& El Mouhayar, 2014; Nilsson \& Juter, 2011; Rivera, 2013; Tanisli \& Özdas, 2009; Walkowiak, 2014; Wilkie, in press). PG is an ability that involves constructing and justifying a well-defined mathematical structure for a given set of initial cues or particular cases. For example, Fig. 1 is a figural pattern with four given stages and with no additional information other than what individual learners infer about its structure that will then be applied to the given stages and beyond.

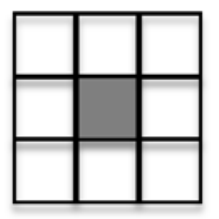

Stage 1

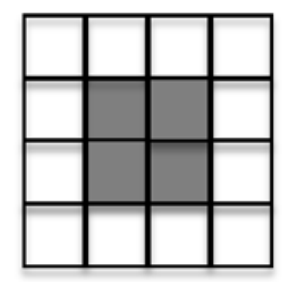

Stage 2

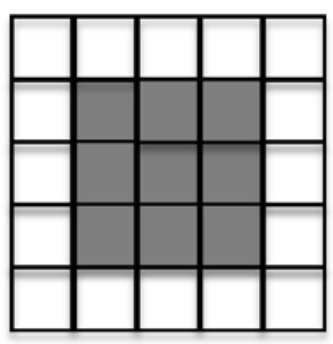

Stage 3

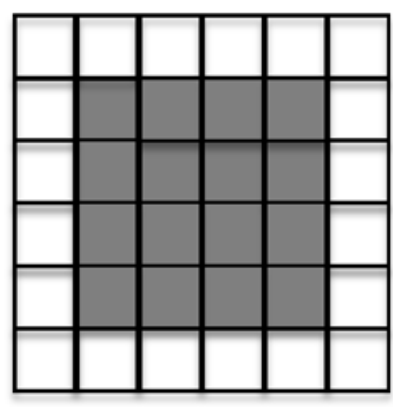

Stage 4

Fig. 1 Square Tiles Pattern

A mathematical structure refers to "a mental construct that satisfies a collection of explicit formal rules on which mathematical reasoning can be carried out" (National Research Council, 2013, p. 29). Hence, PG and mathematical structure are intimately and conceptually intertwined, meaning to say that PG ability is interpretive and rule-driven in nature and enables learners to employ predictive and inferential reasoning despite the initial constraint of having only an incomplete knowledge of the target objects for generalization (e.g., stages in a pattern such as those shown in Fig. 1 or a set of particular instances, situations, or cases). Consequently, every pattern has to be well-defined, that is, any stage, cue, or instance must either exhibit the structure 
or not exhibit the structure. For example, Fig. 2 shows three different but equivalent studentgenerated structures for the Squares Tiles Pattern in Fig. 1.

Che (age 12 years): " $W=4 n+4$. So there's like 4 squares and then you add 4 to each one [corner]. ... And for patio \#2, $2 \times 4$ is 8 , so 1, 2, 3, .., 8, then you add 4. [There are] 4 groups of n.”
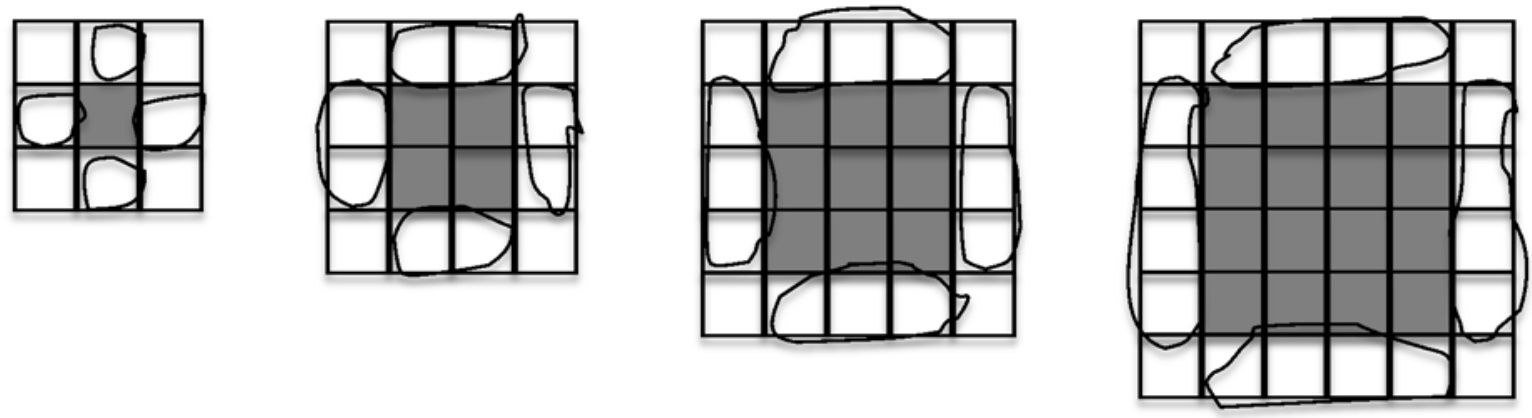

Dina (age 12 years): “4(n+1) coz patio \#1 you have two groups of 4, patio \#2, 3 groups of 4, etc."
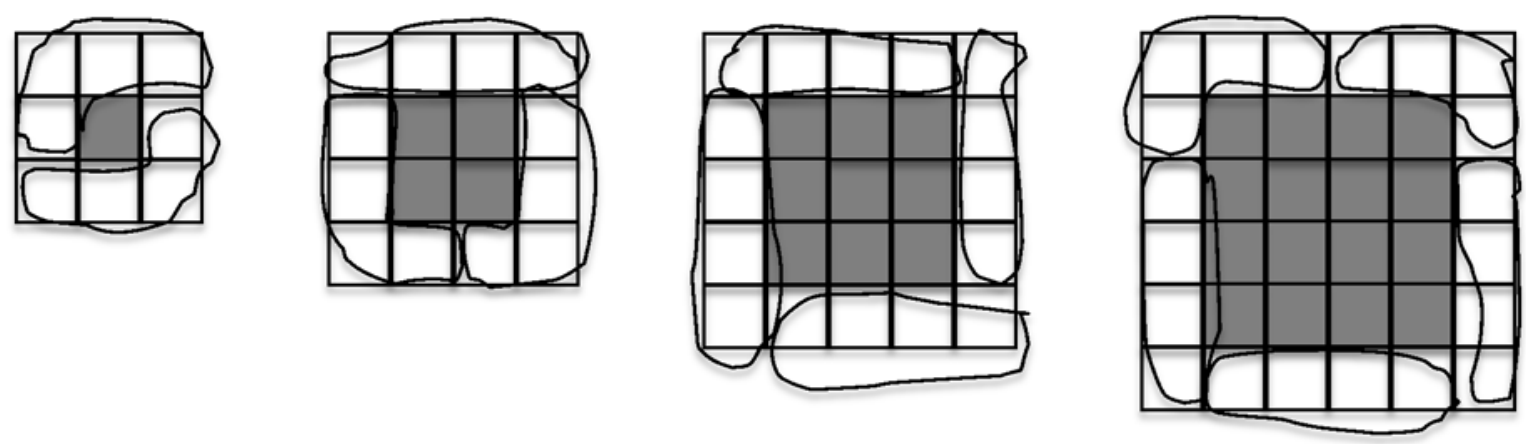
Dave (age 12 years): " $T=2(n+2)+2 n$. The top part, $2+1=3$. Then I multiplied by 2 , the bottom, so that's 6 . And the $2 n$, so here's 1 [row 2 column 1 square] and 1 here [row 2 column 3 square], etc."
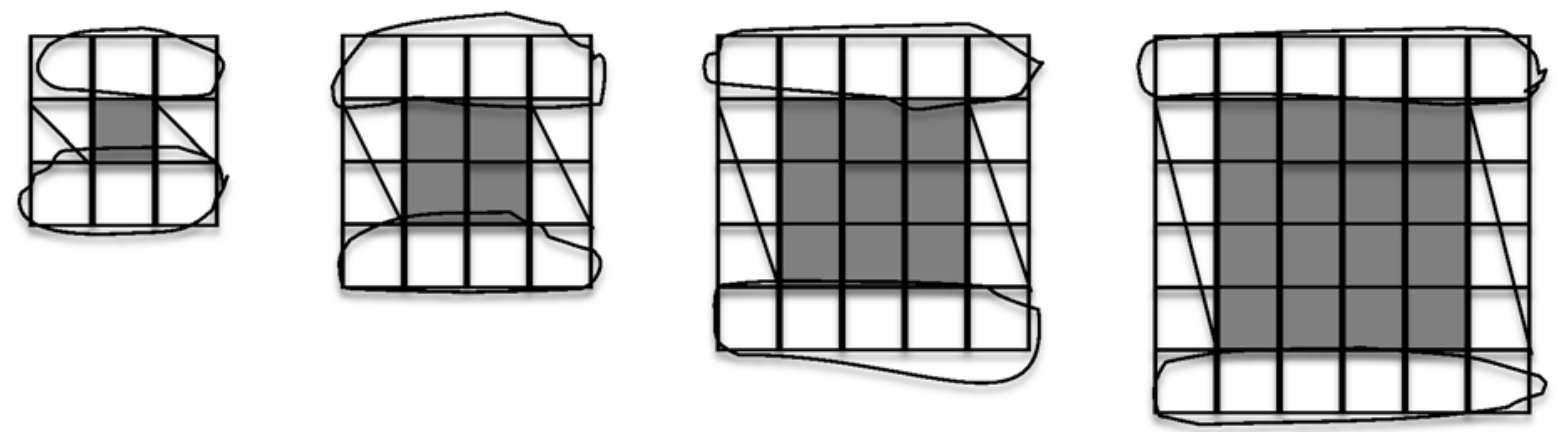

Fig. 2 Grade 8 Student Responses on the Fig. 1 Pattern

On the basis of eighth-grade student Dave's articulated structure, it is easy to infer and predict that stage 235 will have, say, two (top and bottom) rows of 237 unshaded tiles and two (left and right) columns of 235 unshaded tiles surrounding a 235 x 235 shaded square. Furthermore, the students' combined verbal and alphanumeric responses in Fig. 2 convey their respective intensional generalizations for the Fig. 1 pattern, that is, depth-driven statements that express how they analytically visualize the pattern. In Duval's (2014) terms, this visual process is mathematical and involves discerning and recognizing figural units, that is, elements that are mathematically relevant in a given visual representation. We note that the students' statements in Fig. 2 are consequently inductive, which are not causal in nature, and that their inferred structures are abductive, which indicate plausible hypotheses about the pattern. Furthermore, their intensional generalizations help them generate extensional generalizations for the pattern, that is, breadth-driven constructions that have been verified by induction (i.e., in order to construct near stages) and have achieved deductive closure (i.e., the intensional generalization and the known stages together provide the basic hypotheses that yield necessary predictions and outcomes regarding any far stage in the pattern]. ${ }^{\mathrm{i}}$ 
In this paper, we assume that near stages in a pattern refer to stages 9 and below and that far stages deal with stages 10 and above. Near stages enable learners to perform repeated actions or inductive steps involving smaller cases as a way of articulating how they are sensing a general condition through particular stages that they can reasonably manipulate. Far stages require them to employ deductive closure in order to deal with any pattern stage efficiently and effectively without the need to engage in sequential or recursive processing. Any inference made about a particular far stage is an instantiation of the deductively-closed general condition.

The research that is reported in this article was a yearlong investigation that we conducted with a group of 26 US seventh- and eighth grade Algebra I students who were asked to obtain PGs on the two semi-free tasks shown in Fig. 3 before and after a teaching experiment on patterns that emphasized multiplicative thinking. In task 1 of Fig. 3, the students constructed their own near stages by combining two of the three given figural cues. In task 2 of Fig. 3, they constructed near stages from a given initial near stage. 
Task 1. From the following three figures below, pick at least two figures to create a pattern sequence of five stages. Use the attached grid paper to draw your four additional stages.

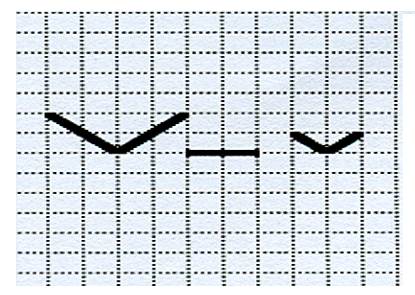

1. What stays the same and what changes in your pattern?

2. Obtain a generalization for your pattern either by describing it in words or by constructing a formula. How do you know that your generalization works?

Task 2. Obtain two different pattern sequences with the following Stage 1 below.

Stage 1

My First Pattern:

Stage 2

Stage 3

Stage 4

Stage 5

General Description or General Formula and Why it Makes Sense

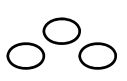

Stage 2

$\underline{\text { My Second Pattern: }}$

Stage 3

Stage 4

Stage 5

General Description or General Formula and Why it Makes Sense

\section{Fig. 3 Two Semi-Free Construction Tasks on a Homework Assessment in Condensed Form}

The two tasks in Fig. 3 are semi-free due to the stipulated design-driven constraints that the students had to fulfill, but they share the same intent as the free generalization tasks of the type Dörfler (2008) recommends. In a commentary critiquing the state of PG research in school contexts through 2008, Dörfler pointed out the following commonplace assumption of "an already prescribed general structure" (p. 153) in almost all patterns given to students:

All students essentially choose the "expected" direction of generalizing, of course with various methods and with different successes. .... Apparently, the statement and the presentation of the tasks, foremost of (growing) linear patterns, have a strong regulating or even restrictive impact on the thinking of the students. The stated questions intimate 
that there is one and only one way to continue the sequence.... The problems given to the students in virtually all papers from this research field not only guide them very strongly but also the figural cues and their arrangement almost exclude trials for other generalizations or continuations. (Dörfler, 2008, p. 153).

Dörfler then suggested the need for all students to engage in open or free generalization tasks, that is, those that are not restricted by pre-given purposes set by the curriculum. Indeed, when such tasks are pursued, they open the door for many interesting PG questions. In this paper, we aim to investigate Dörfler's intriguing suggestion by providing students open or semi-free tasks to determine where sources of the PG constructive process lie. We ask whether the development of mathematical structure in patterning contexts depends on whether students see whole or part configurations. In addition, we ask whether multiplicative thinking plays an important part in PG construction, and whether multiplicative thinking is sufficient for students to process complex patterns of various types.

\section{Emergence of Structures in Everyday and Mathematical Contexts: A Theoretical}

\section{Framework}

\subsection{From Part-to-Shape to Part-to-Relation Structures}

When the 18-to-24-month-old children in Smith's (2003) study were presented with two matched sets of objects, where the first set consisted of examples that fully illustrated their characteristics and the other set displayed only their corresponding caricatures that basically captured their minimal shape features, only the older children could recognize the same representations in both contexts. Among adults, however, object recognition involves inferring interpretive relationships between component parts and wholes. Smith's (2003) synthesis of object recognition involving objects with minimal features also indicates "a general shift from 
more part-like to more configural representations [that occur] with age and with growing expertise in a perceptual domain" (p. 2). Furthermore, a noticeable relational phase emerges when individuals begin to attend to the structural relationships of the components themselves, which has been noted to interact with their developing expertise in a domain. Thus, such studies underscore a conceptual change in object recognition, from part-to-shape features to part-torelations. Applied to middle-school level PG, in this study we initially investigated whether the students' constructed stages relative to the two semi-free tasks shown in Fig. $\mathbf{3}$ reflected this evolved expertise.

\subsection{Articulating Partonomic Relationships in Mathematical Terms}

Two representational mechanisms that are central to PG involve processing and conversion (Duval, 1999), which are sensitive to individual learners' constructed dimensions relative to their emergent patterns. Conversion addresses translation issues in two different representational media or registers (e.g., from the verbal or pictorial to the alphanumeric), while processing addresses transformational issues within the same medium or register (e.g., using properties of operations to simplify expressions). Dimensions refer to levels or numbers of invariant relations, stable changes, or local and global properties and attributes in a pattern. While there is no prescribed minimum or maximum number of dimensions, further processing oftentimes yields equivalent situations that have minimal dimensions.

Among figural patterns, the interpreted shapes of individual stages can also influence the discernment and construction of partonomic properties, and pattern stages that have complex shapes oftentimes require inferring several dimensions and, thus, necessitate a higher cognitive load than pattern stages with simpler shapes. Partonomy is a type of conceptual processing in terms of part-whole relationships; it is also called meronomy, which is a type of conceptual 
structuring that is used frequently in linguistics and computer science (cf. Tversky \& Hemenway, 1984). ${ }^{\text {ii }}$ Animals and their body parts and countries that consist of cities, neighborhoods, and families exemplify nonmathematical instances of partonomic structures. Applied to PG, we note that stable partonomic generalization structures involve making "repeated division into components" (Jüttner, Caelli, \& Rentschler, 1997, p. 245) within and across the constructed stages in any pattern.

In PG, grasping partonomic complexity in stable terms is especially salient in the conversion phase (see Duval (1999) for an example of processing and conversion in geometric contexts). To illustrate, Rivera (2011) reported an interesting incident among his 26 US Algebra 1 students who found the pattern in Fig. 4 more difficult to generalize than the pattern in Fig. 5. The two tasks were administered separately in class over one week as part of a graded assessment task.

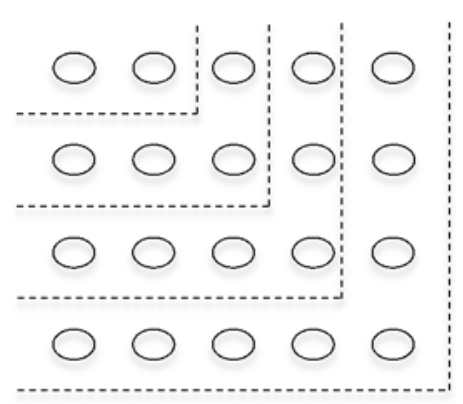
A. How many circles are there in the fifth rectangle? How do you know for sure?
B. Find a direct formula for the number of circles in the nth rectangle. Explain how you obtained it.
C. How many circles are there in the $100^{\text {th }}$ rectangle? How do you know for sure?

\section{Fig. 4 Embedded Rectangular Pattern Generalization Task}


Below are the first four stages in a growing pattern.

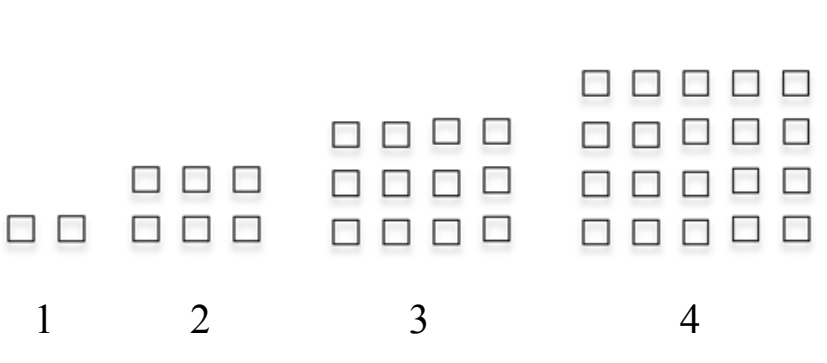

A. Describe stage 10 in a way that makes sense to you (e.g. draw).

B. Find a direct formula that gives the total number of squares $\mathrm{S}$ at any stage $\mathrm{n}$. Explain why your formula is true.

C. Is 9,900 a rectangular number? Explain your answer.

Fig. 5 Separated Rectangular Pattern Generalization Task

PGs for Fig. 4 yielded 65\% correct responses, while PGs for Fig. 5 produced 82\% correct responses. It seemed that the manner in which the Fig.4 figural pattern was presented to the students (e.g., inclusion of segments, embedded nature of the first four stages in one single visual representation) prevented the unsuccessful ones from processing partonomic configurations that really mattered.

\subsection{Absence of Partonomy: Additive Prestructural Mode of Empirical Counting}

Recent PG research results (e.g.: Cadez \& Kolar, 2015; Jurdak \& El Mouhayar, 2014; Nilsson \& Juter, 2011; Rivera, 2013; Tanisli \& Özdas, 2009; Walkowiak, 2014; Wilkie, in press) underscore different abductive partonomic structures on figural patterns. Consider, for instance, the unshaded square tiles in Fig. 1. Many elementary and middle school learners would begin the process of generalizing the total number of unshaded squares by counting them one at a time per stage with little to no conscious awareness of an abductive structure for the pattern. Using a counting-all strategy, some of them might say, "stage 1 would have $1,2, \ldots, 7,8$ squares, stage 2 would have $1,2, \ldots, 11,12$ squares, stage 3 would have $1,2, \ldots, 15,16$ squares, and stage 4 would have $1,2, \ldots ., 19,20$ squares." This type of thinking is additive prestructural since the unshaded squares are seen as single objects with no detectible figural units that might convey a 
meaningful partonomic relationship. Additive prestructural thinking about patterns reflects a mode of empirical counting that is simply meant to answer how many objects are in a given stage; it is practical but is cumbersome and not partonomic. Furthermore, the arithmetical procedure of counting all, which explains the term additive, is the dominant cognitive action underlying the patterning process. There is no generalization at this stage, which explains the term prestructural.

\subsection{Early and Nonstandard ${ }^{i i i}$ Partonomic Structures: Additive Composite (AC) Thinking}

When a counting-all strategy, which uses addition as the primary operation, is replaced by a counting-on strategy, an early form of multiplicative-based generalization emerges. For example, the response "you start at 8 unshaded squares and keep adding 4 unshaded squares" exemplifies an additive composite (AC) partonomic structural thinking; a composite unit ("keep adding 4") is acknowledged as an abductive partonomic component that is scaled in some stable manner from one pattern stage to the next and, thus, becomes the basis for a near stage induction (initial) and a far stage extension (later). AC generalizations are expressed either as early recursive relations or function-based nonstandard formulas or expressions. For example, the expression "keep adding 4 unshaded squares" in relation to the Fig. 2 pattern is recursive; it conveys a minimally partonomic structure but is not useful and efficient in the long term. The function-based nonstandard formula $T(n)=(n+2)+n+n+(n+2)$, where $T$ represents the total number of unshaded squares at every stage number $n$ in Fig. 1, exemplifies a partonomic structural union of four figural units or dimensions, namely, $(n+2)$ unshaded squares on the top row, $n$ unshaded squares on the left column, $n$ unshaded squares on the right column, and $(n+2)$ unshaded squares on the bottom row. 
A contracted multiplicative (CM) partonomic structural thinking about the pattern in Fig. $\mathbf{1}$ is a standard equivalent version of all function-based nonstandard $\mathrm{AC}$ generalizations that consequently involves a minimal number of dimensions. For example, Dave's nonstandard AC generalization in Fig. 2 consists of 4 dimensions, unlike Che's standard generalization that involves only two dimensions, namely, the stage number represented by the $n$ unshaded middle squares around the sides and the constant set of 4 unshaded corner squares. Che's formula $W(n)$ $=4 n+4$ in Fig. 2 exemplifies a contracted partonomic structure, which is different from the one that Dave produced that could still be converted and processed into its equivalent standard form.

In the Algebra 1 class that participated in the yearlong study and teaching experiment, the students dealt with PG tasks that supported their transition from mere empirical counting in additive prestructural terms to early/recursive, nonstandard, and contracted/standard partonomic multiplicative thinking about their patterns. In Experiment 1, we investigated whether the experiment had a strong influence on both cohorts' PG ability by assessing the extent to which their partonomic structures evolved from additive prestructural to AC and $\mathrm{CM}$. We also assessed the extent to which their multiplicative thinking ability was sufficient in helping them convert their verbal figural-driven PG processing in alphanumeric form.

\section{Rationale of the Study}

In this study, we compared the performances of two cohorts of students (mean age of 12.5 years) that came from the same Algebra 1 class in an urban school in Northern California (USA). The students in Cohort I ("experts"), who were in eighth grade at the time of the study, had already participated in an earlier two-year study on PG with the authors. The students in Cohort II ("novices") consisted of seventh and eighth graders who were added to the Algebra 1 class at the beginning of the school year. While the novices did not formally explore PG in the 
earlier years, both cohorts were exposed to the same mathematics curriculum, resources, and assessments in Algebra 1. The two cohorts were also equivalent on the basis of their pre-algebra performances on the state assessment, including race profile and level of socioeconomic status.

Having two different cohorts, one considered expert and the other novice in PG, enabled us to compare their PG ability on a novel mathematical task, that is, none of them had formal experiences with (semi-) free patterning tasks in the earlier years. In terms of possible research implications, it is likely the case that they would exhibit different PG performances in either processing or converting phases. Expert-novice studies in the general pattern recognition literature have, indeed, indicated differences (e.g.: Bilalic et al. (2010); Behrmann \& Ewell (2003); Larkin et al. (1980)). Expert patterners, in particular, have been documented to possess an "enhanced specificity that accompanies increasing experience," which means that the manner in which they would construct configural content involves a type of simultaneous processing of individual features and relationships among them (Behrmann and Ewell, 2003, p. 480). In figural PG contexts, we thought that it would be interesting to determine similarities and differences in the two cohorts' processing and conversion of emergent structures before and after a teaching experiment since the experiment provided both cohorts with mathematical experiences in developing a partonomic multiplicative thinking ability.

Thus, in this study, we address the following research questions:

(1) What are some sources of the abduced structures exhibited by seventh and eighth grade students (mean age of 12.5 years) in the context of (open) semi-free PG tasks?

(2) Does the emergence of a mathematical structure in PG depend on whether students see the initial step in patterning either as a whole unit or as a configuration among parts (i.e., mathematically thinking about dimensions in an emerging pattern in terms of wholes or 
parts, respectively)? Furthermore, what role does having a multiplicative thinking disposition toward mathematical structures play in PG conversion and processing?

(3) Is multiplicative thinking sufficient to process various types of patterns and support the conversion of interpreted structures into function-based generalizations?

Experiment 1 deals with issues (1) and (2), and Experiment 2 addresses issue (3).

\section{Experiment 1}

We analyzed the PG performances of both cohorts on the two tasks shown in Fig. 3 before and after the two-week teaching experiment. We wanted to test the following observations that: (1) before the experiment, both cohorts would process more part- than whole-driven growth structures and that the expert group would favor more standard function-based CM generalizations than the novice group who would have a preference toward early/recursive and nonstandard function-based AC generalizations; and (2) after the experiment, both cohorts would exhibit about the same or similar expert levels of PG performance.

\subsection{Participants}

Before the teaching experiment, twenty-six students (22 Southeast Asian, 2 South Asian, 1 White, and 1 African American; 17 females and 9 males) submitted responses on the two tasks. After the experiment, sixteen students (13 Southeast Asian, 2 South Asian, and 1 White; 10 females and 6 males) submitted responses on the same tasks. They received the same intervention at the same time and the tasks were given as homework so that they would have ample time to accomplish them ${ }^{\text {iv }}$.

\subsection{Teaching Experiment, Tasks, and Phases}

Prior to the teaching experiment, the first author, instructor for the class, solicited volunteers to work on the two tasks. The students received bonus points for submitting their 
work and were reminded to accomplish the tasks independently. The first author used bonus points frequently as a classroom practice to help them make up for lost or missing points in their other assignments. Hence, the students did not perceive the bonus incentive on the homework tasks to be novel enough that it might have affected the outcomes of the experiment in significant ways. During the teaching experiment, which took place two weeks after the official start of classes, the classroom activities focused on linear PG. Linear PG introduced the students to relevant concepts that mapped to concepts and processes involving linear functions and modeling, which were major state standards in the Algebra 1 curriculum. In the beginning phase of the experiment, they reviewed the concept of multiplication of two whole numbers, where $a \times b$ pertained to the total number of objects in $a$ groups of $b$ elements each. ${ }^{\mathrm{v}}$ They learned to partition whole sets of objects in ways that employed a figural unit as a starting point. They visually interpreted such units in terms of shapes that could be scaled by repetition and reconfiguration. Fig. 6 illustrates three different student-generated partonomic processing of the cross dots problem in multiplicative terms. 
$(2 \times 6)+(2 \times 6)-(2 \times 2)$

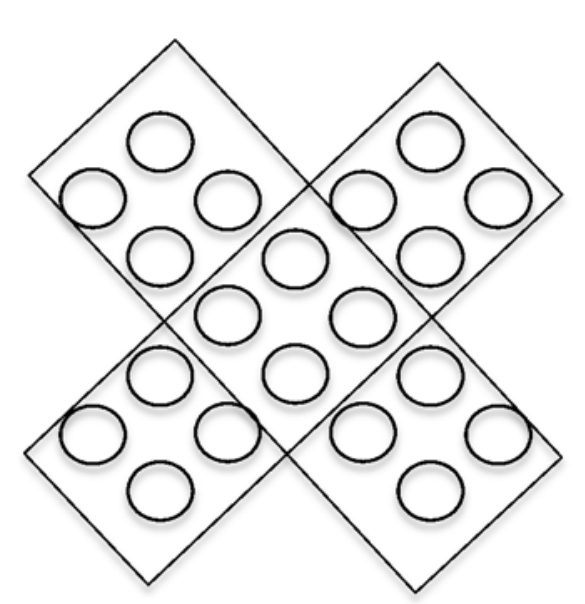

$4 \times 5$

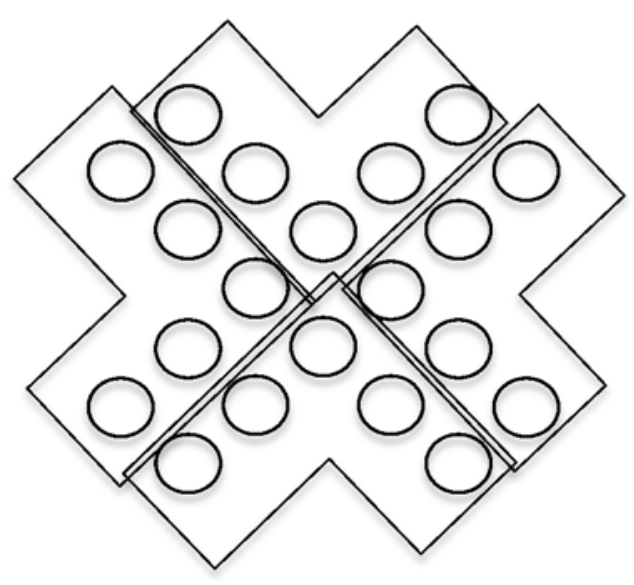

$5 \times 4$

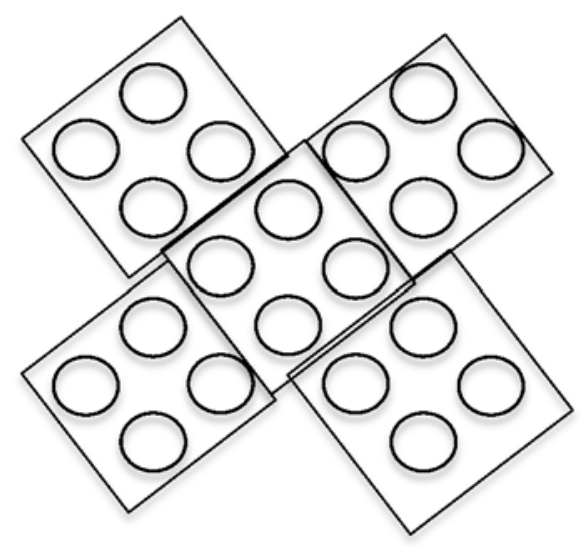

Fig. 6 Student Work on the Cross Dots Problem

The converted expression $4 \times 5$ meant constructing 4 groups of 5 circles each, where each group takes the shape of a boomerang. The remaining two expressions emerged after inferring symmetry on the set of circles. The students also learned that converted arithmetical expressions could arise from seeing equivalent partonomic structures of the same set. They then worked on sequential-based linear PG tasks (e.g., the consecutive four stages in Fig. 1) and toward the end of the experiment dealt with non-sequential-based linear PG (e.g., stages 2 and 4 only in Fig. 1). 
Several days after the closing of the teaching experiment, the first author solicited volunteers once more to work on the same two tasks in Fig. 3 as homework.

\subsection{Coding}

We coded the before and after sets of responses separately. Only correct PG responses were coded in detail and analyzed by cohort. The incorrect responses reflected sequences of stages that did not convey well-defined mathematical structures and, thus, were not sufficiently meaningful and worthwhile to analyze. Correct figural growth structures were categorized either as whole stage-driven or part-driven and the corresponding converted expressions were then classified as early/recursive relation AC, nonstandard function-based AC, or standard functionbased CM. Each correct response was assigned 1 point, and interrater agreement was 92\%. We illustrate our coding process using the two sets of student-generated patterns shown in Figs. 7 and 8.

Fig. 7 shows three examples of whole stage-driven patterns, where the same whole unit of 3 circles (i.e., stage 1) is iterated in three different ways. In the case of Shawna and Emma, the abduced structure of seeing groups or multiples of 3 circles is converted into and processed as standard function-based CM formulas. Kenny's PG, however, is interesting. While he sees his pattern in early/recursive AC terms (keep adding 3 circles at every stage), his converted generalization in alphanumeric form is processed as a standard function-based CM formula $(y=3 s)$.

\section{Shawna (Cohort 1, Grade 8)}
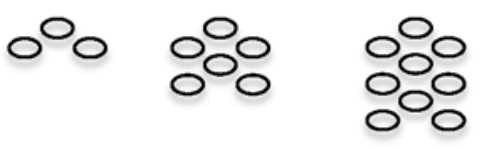

3

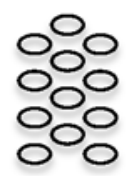

4

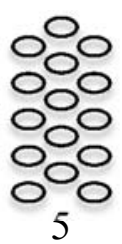

$P=n \times 3$. The stage number multiplied by three equals the amount of circles. $n$ means stage number. 


\section{Emma (Cohort 1, Grade 8)}

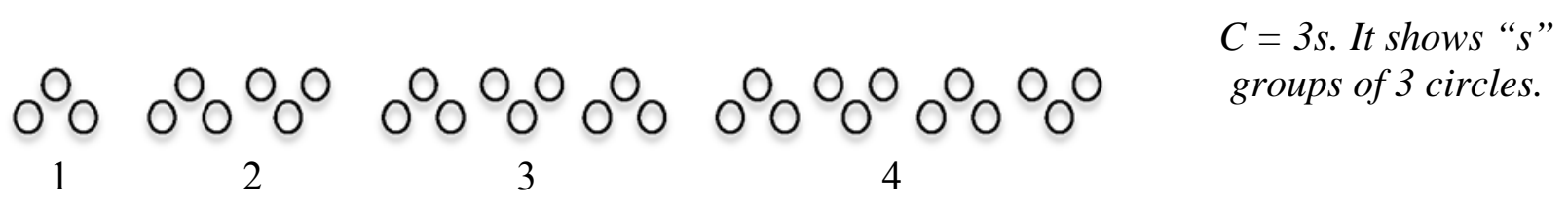

Kenny (Cohort 1, Grade 8)

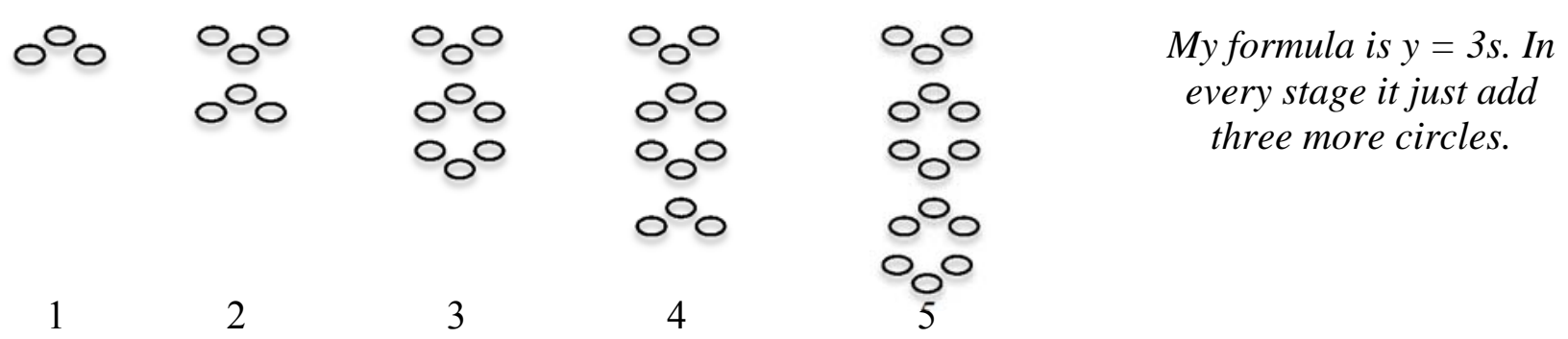

Fig. 7 Examples of Expert Whole-Stage Linear Growth Patterns Relative to Fig. 3 Task 2

Fig. 8 shows two examples of part-driven patterns. Jake's pattern consists of three sides that increase at the same time according to stage number, while Selma's pattern has a structure with a fixed top circle and two corners that grow by the stage number. Jake's case illustrates a nonstandard function-based AC thinking about the pattern (three sides each with $n$ circles) that is then converted into and processed as a standard function-based CM formula $(p=n \times 3)$. Selma's case illustrates an early/recursive AC thinking about the pattern (adding 2 circles each time, one on each corner) that does not further transform in both aspects of conversion and processing into either a nonstandard function-based AC (e.g., $1+2,1+2+2,1+2+2+2,1+2+2+2+2$, $\ldots, 1+n$ number of $2 \mathrm{~s}$ ) or a standard function-based CM (e.g., $1+n \times 2$ or $1+2 n$ ). 


\section{Jake (Cohort 1, Grade 8, Dimension 1)}

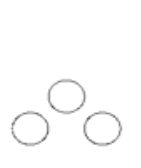

1

$\mathrm{OO}$

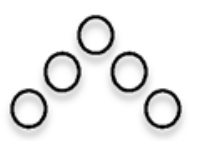

2

1

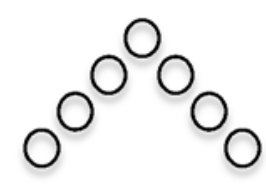

3

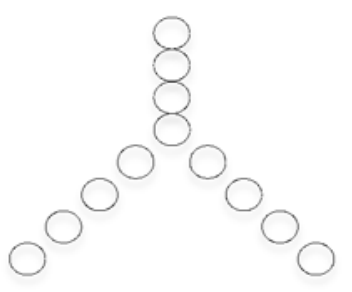

4 $p=n \times 3$. By multiplying $n$

by 3 equals the answer. There's three sides and the amount of circles on each side is $n$.

\section{Selma (Cohort 2, Grade 7, Dimension 2)}

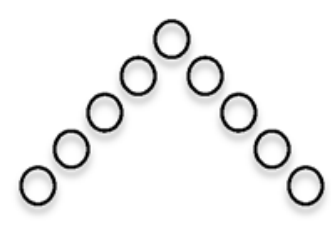

4

Fig. 8 Examples of Part-Driven Linear Growth Patterns Relative to Fig. 3 Task 2

\subsection{Results}

\subsubsection{Part-Driven Abductive Structures}

Tables 1 and 2 provide summaries of the students' abductive structures organized by cohort and type of structure before and after the two-week teaching experiment, respectively. Before the experiment, about $69.5 \%$ of the responses in both cohorts favored part-driven structures in comparison with about $19 \%$ that preferred whole stage-driven structures. Those students who generated patterns with part-driven structures usually extended parts or sides of their initial stages in a consistent and stable manner, yielding similar figures that grew according to their stipulated abductions. After the experiment, about $70.5 \%$ of the responses continued to favor part-driven structures over about $27.5 \%$ that preferred whole stage-driven structures. There was, however, a significant increase in the percentage of responses that favored whole stagedriven structures after the teaching experiment. 


\begin{tabular}{|c|c|c|c|}
\hline Type of Structure & $\begin{array}{c}\text { Cohort 1 (Expert; } \\
\mathrm{n}=13)\end{array}$ & $\begin{array}{c}\text { Cohort 2 (Novice; } \\
\mathrm{n}=13)\end{array}$ & $\begin{array}{c}\text { Average Across } \\
\text { Cohorts }\end{array}$ \\
\hline Whole stage-driven structures & .15 & .23 & .19 \\
\hline Part-driven structures & .72 & .67 & .695 \\
\hline Incorrect responses & .13 & .10 & .115 \\
\hline
\end{tabular}

Table 1 Students' Abductive Structures Before the Teaching Experiment Relative to the Fig. 3 Tasks

\begin{tabular}{|c|c|c|c|}
\hline Type of Structure & $\begin{array}{c}\text { Cohort 1 (Expert; } \\
\mathrm{n}=7)\end{array}$ & $\begin{array}{c}\text { Cohort 2 (Novice; } \\
\mathrm{n}=9)\end{array}$ & $\begin{array}{c}\text { Average Across } \\
\text { Cohorts }\end{array}$ \\
\hline Whole stage-driven structures & .33 & .22 & .275 \\
\hline Part-driven structures & .67 & .74 & .705 \\
\hline Incorrect responses & .00 & .14 & .07 \\
\hline
\end{tabular}

Table 2 Students' Abductive Structures After the Teaching Experiment Relative to the Fig. 3 Tasks

The three examples of student work in Fig. 9 illustrate part-driven growth structures that were constructed after the teaching experiment. Dung's tower pattern had a base of two circles that stayed the same at every stage and a vertical column of circles that grew according to stage number. Jana's W-pattern had a fixed circle in the middle top portion and two equal sets of circles configured symmetrically and whose cardinality matched the stage number. Delilah's triangular pattern had two parts consisting of segment-sides and middle segments that grew according to the nonlinear rule that she stipulated for each part. For the segment-sides, the expression $4 x$ meant seeing four groups of the stage number $x$. In the case of the middle segments, the expression $x(x-1)$ meant seeing $x$ groups of $(x-1)$ sticks.

\section{Dung (Cohort 1, Grade 8)}

1

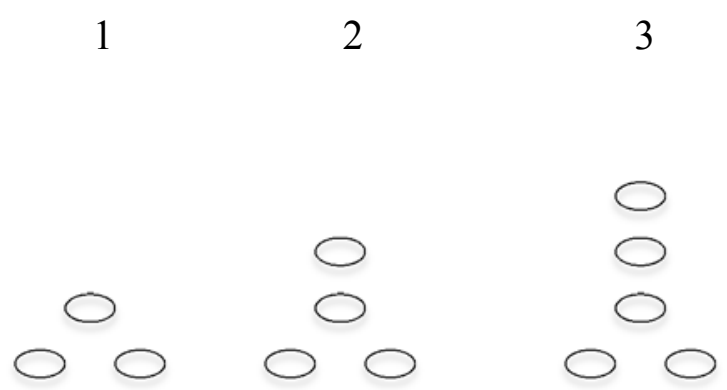

4

5

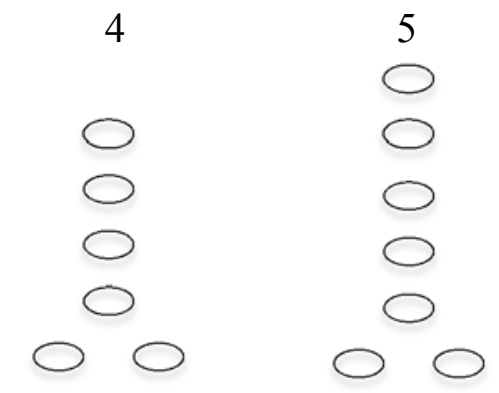

$$
C=n+2
$$




\section{Jana (Cohort 2, Grade 7)}

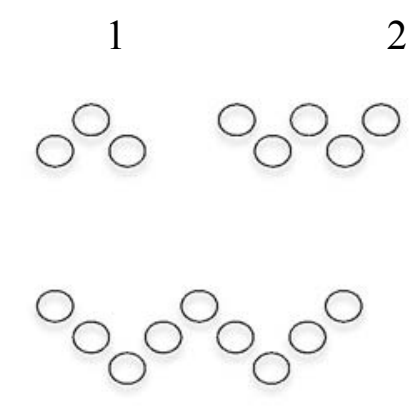

4
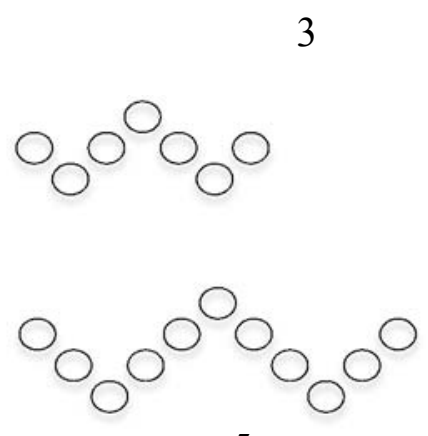

5
$y=2 s+1$. Two groups of the stage \# plus the one on top

\section{Delilah (Cohort 2, Grade 7)}

Number 1 will stay in all of them. $L=x(x-1)+4 x$ can be simplified to $x^{2}+3 x$. The $x(x-1)$ is for the lines in the middle of the triangle. The $+4 x$ is for the [lines along the] triangle borders.

It's really short for $x^{2}+3(x)$.

Fig. 9 Examples of Part-Driven Linear and Nonlinear Growth Structures Constructed After the Teaching Experiment 
Across the two cohorts, stable growth in a figural pattern meant adding the same number of pieces (e.g., segments or circles) along the same location(s) or part(s) within each constructed figure. Furthermore, the constructed stages together resembled dilated growing figures. No similitude constructions were produced. An example of a similitude construction is shown in Fig. 10, a variation of Selma's part-driven growth structure shown in Fig. 8. Such stages could be perceived as involving a combination of rotation and dilation. This particular point about dilated and similitude constructions is worth noting in light of documented work with younger children who tend to produce both types of patterns and yet do not see them as equivalent (Rivera, 2013).

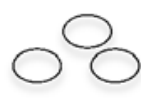

1

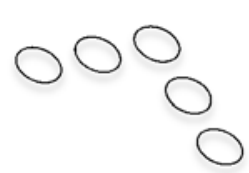

2

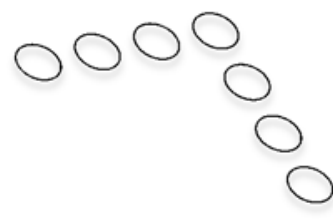

3

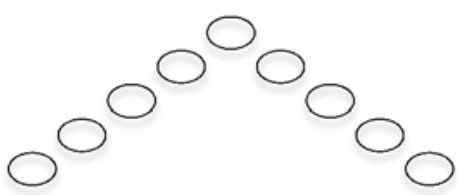

4

Fig. 10 A Similitude Construction of Selma's Dilated Pattern in Fig. 8

\subsubsection{Whole Stage-Driven Abductive Structures}

Among the novice cohort, they constructed about the same number of patterns that reflected whole stage-driven structures before and after the experiment (about 22.5\%). Among the expert cohort, the number of such patterns increased from about $15 \%$ to $33 \%$. Across the two cohorts, however, there was a noticeable increase in the number of patterns with whole stagedriven structures from before to after the experiment (i.e., from about $19 \%$ to $27.5 \%$ ). Whole stage-driven structures from both cohorts reflected three categories of patterns, namely: oscillating; cyclic-linear growth; and repeating-linear growth. Fig. 11 shows Ollie's oscillating 
pattern on the first task, where "every odd numbered stage takes up 2 squares" and "every even numbered stage takes up 4 squares.”

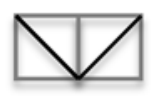

1

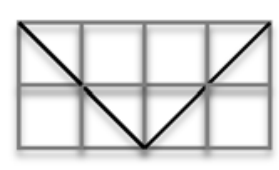

2

Ollie (Grade 7, Cohort 1)

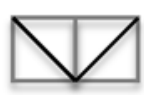

3

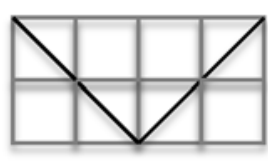

4

In every odd numbered stage, the figure takes up 2 squares. In every even numbered stage, the figure takes up 4 squares. It works because it increases every even numbered stage and decreases in every odd numbered stage.

\section{Fig. 11 An Example of a Whole Stage-Driven Oscillating Pattern Before the Teaching Experiment}

Fig. 12 shows Dina's 3-cyclic linear growth pattern, where stages 1, 2, and 3 represent a full cycle that repeats after every set of 3 consecutive stages. In her written description of the pattern, she infers that "for stage 1, it is already given, so for stage 2, you go right and then down, [and] for stage 3, you go right and then up afterwards - the pattern starts over again afterwards." 


\section{Dina (Grade 8, Cohort 2)}

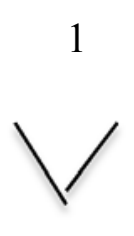

2

3

4
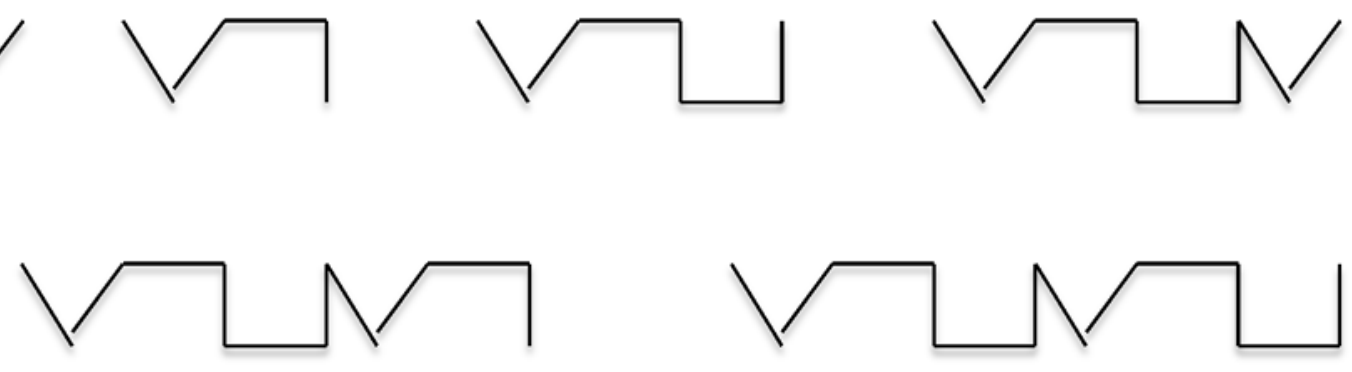

5

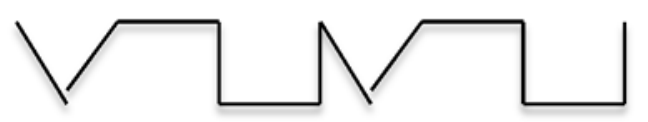

6

The lines are the same length although the only things different are what shape they are in. For stage 1, it is already given, so for stage 2, you go right and then down! For stage 3, you go right and then up afterwards. The pattern starts over again afterwards.

Fig. 12 An Example of a Whole Stage-Driven Cyclic-Linear Growth Pattern Before the Teaching Experiment

Figs. 13 and 14 show two examples of repeating-linear growth patterns. In Fig. 13, Dung's stage 1 consists of a V shape adjoined by a horizontal segment on its vertex. Stage 2 then consists of two copies of stage 1, stage 3 has 3 copies of stage 1, etc. His explicit expression, however, conveys a relationship involving the two parts or dimensions that comprise his stage 1 , that is, $\mathrm{nV}+\mathrm{n}$

\section{Dung (Grade 8, Cohort 1)}

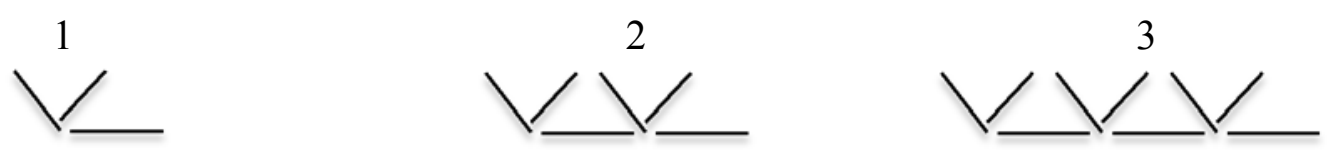

4

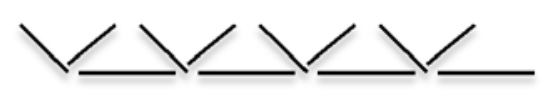

5

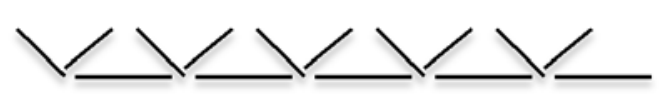

$\mathrm{nV}+\mathrm{n}$

Fig. 13 An Example of a Whole Stage-Driven Repeating-Linear Growth Pattern Generalized to a Recursive Formula Before the Teaching Experiment 
In Fig. 14, Tamara's stage 1 consists of a $\mathrm{V}$ shape and a horizontal segment that together form one triangle. Her stage 2 has 2 adjacent triangles that have been adjoined to the stage 1 triangle. Her stage 3 has 5 adjacent triangles, stage 4 has 7 triangles, and so on. Tamara's structure involves "adding 2 [triangles] each time," which explains her converted formula $T(s)=2 \times S-1$, where $T$ means the number of triangles at every stage $S$.

\section{Tamara (Grade 8, Cohort 1)}

1

$\bigvee$

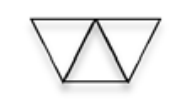

3

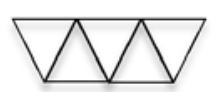

4

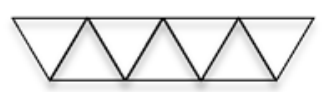

5

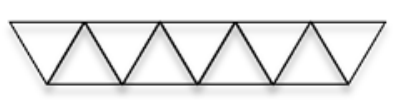

You are adding two [triangles] each time. $2 \times S-1=T$

Fig. 14 An Example of a Whole Stage-Driven Repeating-Linear Growth Pattern Generalized to a Function-Based Formula Before the Teaching Experiment ( $S$ means "stage number" and $T$ means "total number of triangles")

\subsubsection{Converted Representations}

Tables 3 and 4 provide summaries of the students' converted verbal and alphanumeric generalizations organized by type of formula and cohort before and after the teaching experiment, respectively.

\begin{tabular}{|c|c|c|}
\hline Type of Formula & $\begin{array}{c}\text { Cohort 1 } \\
\text { (Expert; } \\
\mathrm{n}=13)\end{array}$ & $\begin{array}{c}\text { Cohort 2 } \\
\text { (Novice; } \\
\mathrm{n}=13)\end{array}$ \\
\hline $\begin{array}{c}\text { Early/Recursive Additive Composite AC } \\
\text { (e.g., Keep adding 4 unshaded squares in the Fig. 1 pattern) }\end{array}$ & .15 & .44 \\
\hline $\begin{array}{c}\text { Nonstandard Function-Based AC } \\
\text { (e.g., T(n) }(\mathrm{n}+2)+\mathrm{n}+\mathrm{n}+(\mathrm{n}+2) \text { in the Fig. 1 pattern) }\end{array}$ & .03 & .05 \\
\hline $\begin{array}{c}\text { Standard Function-Based CM } \\
\text { (e.g., W(n) }=4 \mathrm{n}+4 \text { in the Fig. 1 pattern) }\end{array}$ & .69 & .41 \\
\hline
\end{tabular}

Table 3 Students' Converted Generalizations Relative to the Fig. 3 Tasks Before the Teaching Experiment 


\begin{tabular}{|c|c|c|}
\hline Type of Formula & Cohort 1 (Expert; $\mathrm{n}=7$ ) & Cohort 2 (Novice; $\mathrm{n}=9$ ) \\
\hline Early/Recursive AC & .00 & .08 \\
\hline Nonstandard Function-Based AC & .10 & .00 \\
\hline Standard Function-Based CM & .81 & .78 \\
\hline
\end{tabular}

\section{Table 4 Students' Converted Generalizations Relative to the Fig. 3 Tasks After the} Teaching Experiment

Before the teaching experiment, the expert cohort constructed more standard function-based CM formulas $(\sim 69 \%)$ than the novice cohort ( 41\%). Furthermore, the novice cohort constructed about the same number of early/recursive AC ( 44\%) and standard function-based CM ( 41\%) formulas. After the experiment, both cohorts constructed about the same number of standard function-based $\mathrm{CM}$ formulas ( $81 \%$ and $78 \%$ ). Nonstandard function-based $\mathrm{AC}$ formulas were not too common across the two cohorts before and after the teaching experiment.

\subsubsection{Discussion}

The preceding results suggest that one source of the students' abduced structures was driven by their preference toward constructing and seeing mostly linear growth patterns that generally had at most two dimensions. Furthermore, while patterns with oscillating (e.g., Fig. 11) and 3-cyclic linear growth (e.g., Fig. 12) structures were no longer evident in their responses after the teaching experiment, there was, however, a noticeable increase in (1) whole stagedriven patterns with repeated linear-growth structures among the expert cohort (e.g., Figs. 7, 13, and 14) and (2) part-driven patterns with linear growth structures among the novice cohort (e.g., Figs. 8 and 9).

The whole stage-driven patterns with repeated linear-growth structures in Fig. 7 all have dimension 1 , that is, stage 1 is the one and only figural unit that is used to build the succeeding stages. Consequently, converting their structures from the visual and verbal to the alphanumeric is rather easy to accomplish since the corresponding $\mathrm{CM}$ forms convey direct variations (i.e., linear functions with no constant term). Certainly, not all dimension-1 whole stage-driven 
repeated linear-growth patterns convey direct variations. For example, Tamara's pattern in Fig. 14 involves adjoining pairs of adjacent triangles at every stage number. However, her converted standard function-based CM conveys a linear function with a constant term. If Tamara opted to count the number of sides instead of the number of whole-unit triangles in Fig. 14, the converted nonstandard AC and standard CM formulas would also be linear functions with a constant term. In other words, different ways of seeing and interpreting structures for whole stage-driven repeated linear-growth patterns influence and complicate both aspects of PG conversion and processing.

The most prevalent constructed structures involve part-driven linear growth patterns with dimension $2^{\text {vi }}$. The figural patterns of Selma (Fig. 8) and Jana (Fig. 9) involve coordinating two stable dimensions, that is, a fixed middle circle and two legs that grow consistently (i.e., in both count and spatial location) in a symmetrical manner. The constructions of Dung and Delilah in Fig. 9 also involve coordinating two stable dimensions. Dung's pattern has a fixed base and a column of circles that increases linearly according to stage number. Delilah's nonlinear pattern involves two figural parts that increase along two dimensions, namely, the perimeter segments and the middle horizontal segments. Consequently, the converted forms convey standard function-based $\mathrm{CM}$ formulas involving binomial expressions corresponding to the two dimensions. Furthermore, simple cases of patterns such as those that Jana and Dung constructed in Fig. 9 involve a straightforward conversion from the verbal to the alphanumeric that oftentimes does not require additional processing. In Delilah's case, however, the way she justified her formula for the nonlinear pattern in Fig. 9 illustrates a two-level transformational processing from the initial to the final form. 
The cyclic structure of Dina's pattern in Fig. 12 has dimension 3 because the repeated figural unit is completed over three successive stages of construction. We did not come across other student-generated structures involving 4 or more dimensions. Perhaps indirectly, the tasks that were presented to the students appealed to them as necessitating expected ways of generalizing as a consequence of their classroom experiences with mostly dimension-2 type linear patterns, which could also be another source of their abduced structures.

The teaching experiment on multiplicative thinking significantly influenced the two cohorts' PG processing in both aspects of figural conversion and processing. Tables 1 and 2 indicate that the percentage of incorrect responses from before to after the teaching experiment across the two cohorts decreased. Tables 3 and $\mathbf{4}$ show that the percentage of standard functionbased CM formulas increased significantly with a noticeable decrease in the use of early/recursive AC formulas. Indeed, a multiplicative thinking disposition toward constructing and justifying mathematical structures appears to be a necessary condition in PG conversion and processing. The sufficient condition is explored in section 5.

Findings from Experiment 1 also indicate a remarkable PG conversion and processing of individual student-generated figural patterns having at most 2 dimensions. Furthermore, the semi-free patterning activity provides students with an opportunity to learn how to coordinate figural pattern construction and structure formation and justification in an emerging context. However, two additional issues still need to be clarified, as follows. The first issue involves determining the sustainable impact of the necessary condition. Our assessment of students' PG ability involving semi-free tasks occurred over a short timeframe, which is a typical practice in almost all patterning studies. Consequently, we could not fully ascertain the necessity of multiplicative thinking in supporting the conversion and processing of various kinds and types of 
(figural and numerical) patterns in the long term. The second issue involves understanding how students process (semi-)free patterning tasks in a clinical setting. Given the constraints in which we collected our data in Experiment 1, we did not have access to the manner in which the students personally engaged on such tasks, which would be useful to know in order to clarify and deepen our understanding of the complex relationship between dimension and figural unit construction and PG conversion and processing. We deal with these two issues in Experiment 2.

\section{Experiment 2}

In Experiment 2, we analyzed the PG performances of a subset of 11 students (4 males, 7 females) across the two cohorts four and a half months after the teaching experiment. No explicit PG activity of the type pursued in the first experiment occurred during these months. Each student was clinically interviewed by the first author and given 55 minutes to work on four PG tasks. For the purpose of this particular study, we discuss only the students' responses on the semi-free task shown in Fig. 15, which is a variation of Fig. 3 task 2.

Below are the first two stages in a growing pattern of squares.
$\begin{aligned} & \text { 1. Continue the pattern until stage } 5 . \\ & \text { 2. Find an explicit formula in two different ways. Justify each } \\ & \text { formula. } \\ & \text { 3. If none of your formulas above involve taking into account } \\ & \text { overlaps find a direct formula that takes into account overlaps. } \\ & \text { Justify your formula. } \\ & \text { 4. How do you know for sure that your pattern will continue that } \\ & \text { way and not some other way? } \\ & \text { 5tage } 2 \quad \text { Find a different way of continuing the pattern and obtain a } \\ & \text { explicit formula for this pattern. }\end{aligned}$

Fig. 15 Square Pattern Task in Compressed Form

\subsection{Results}




\subsubsection{Continued Dominance of Dimension-2 Part-Driven Linear Structures}

Fig. 16 shows six different part-driven dimension 2-type student-generated patterns and their converted alphanumeric generalizations relative to the Fig. 15 task. Four of the patterns (i.e., A, B, C, and F) have linear structures, and standard function-based CM formulas were offered more frequently than nonstandard function-based AC formulas. Pattern A was the most favored construction and produced the most number of equivalent function-based $\mathrm{AC}$ and $\mathrm{CM}$ formulas. The four students who constructed nonlinear patterns D and E initially conveyed their structures as early/recursive AC expressions. However, they found it difficult to process them further into their equivalent standard function-based CM versions on their own. The three

students who obtained the same standard function-based CM for pattern D (i.e., $\left.s=n \times(n+1) \frac{1}{2}\right)$ accomplished the task in joint activity with the interviewer. 


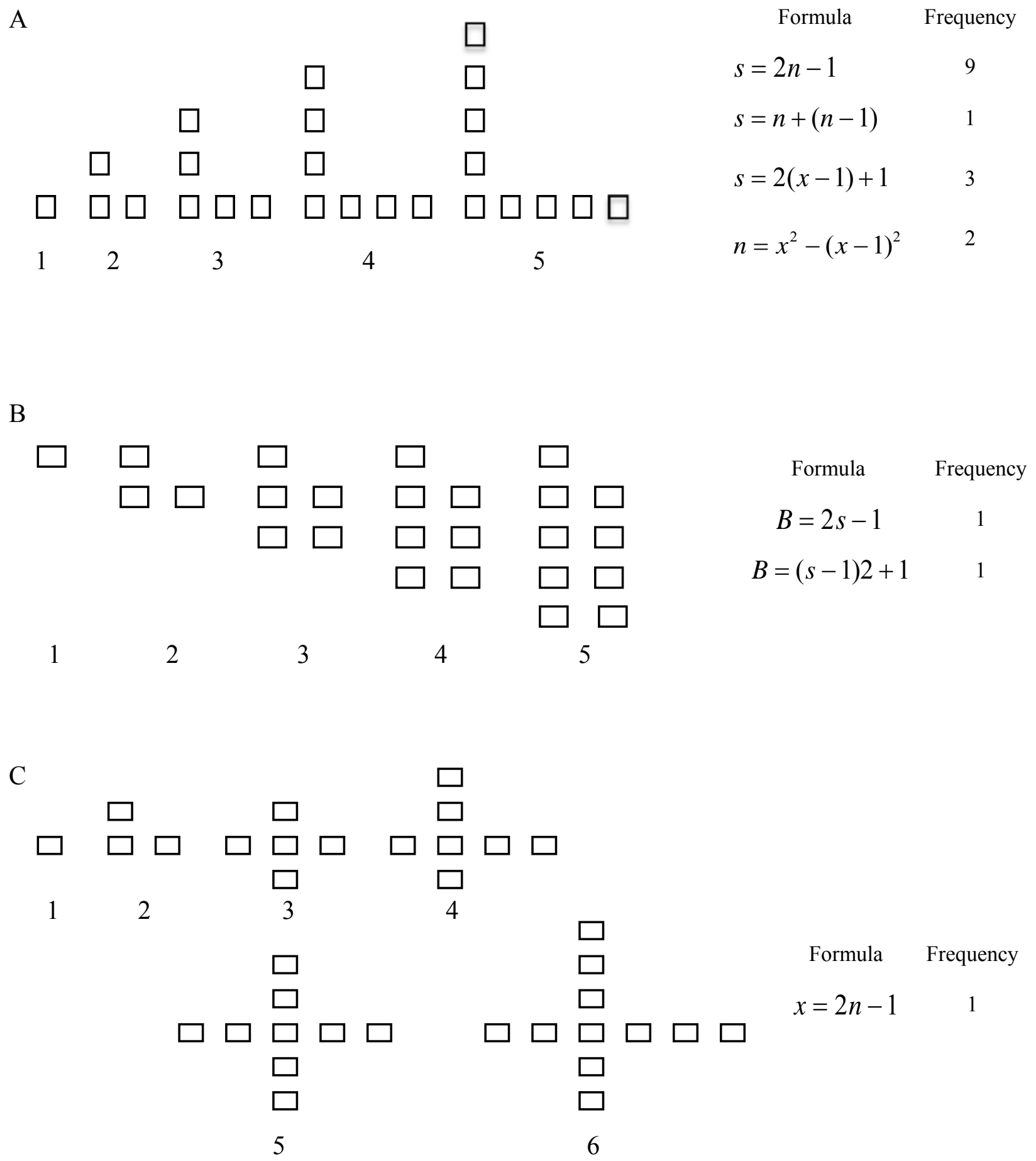



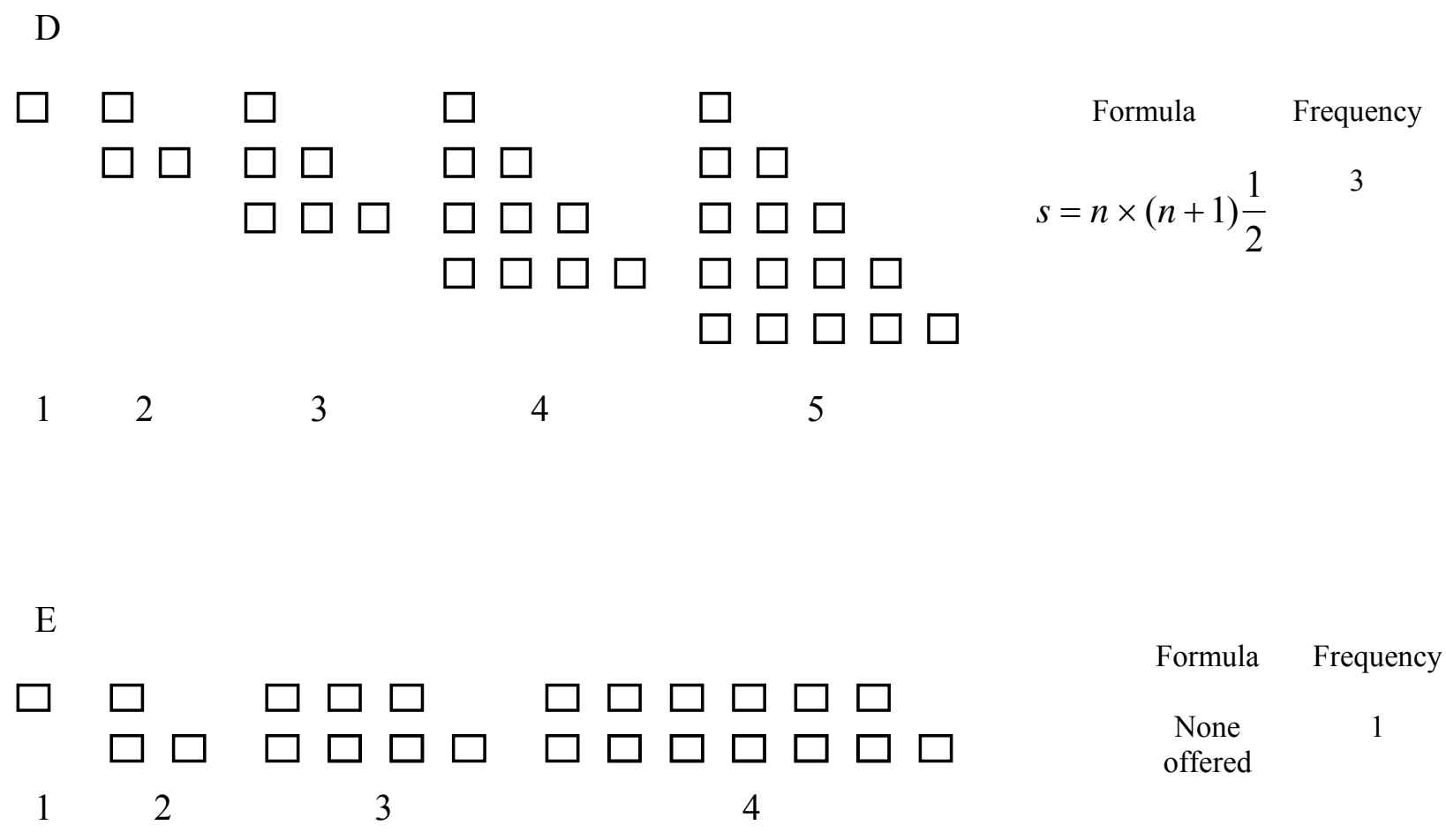

$\mathrm{F}$

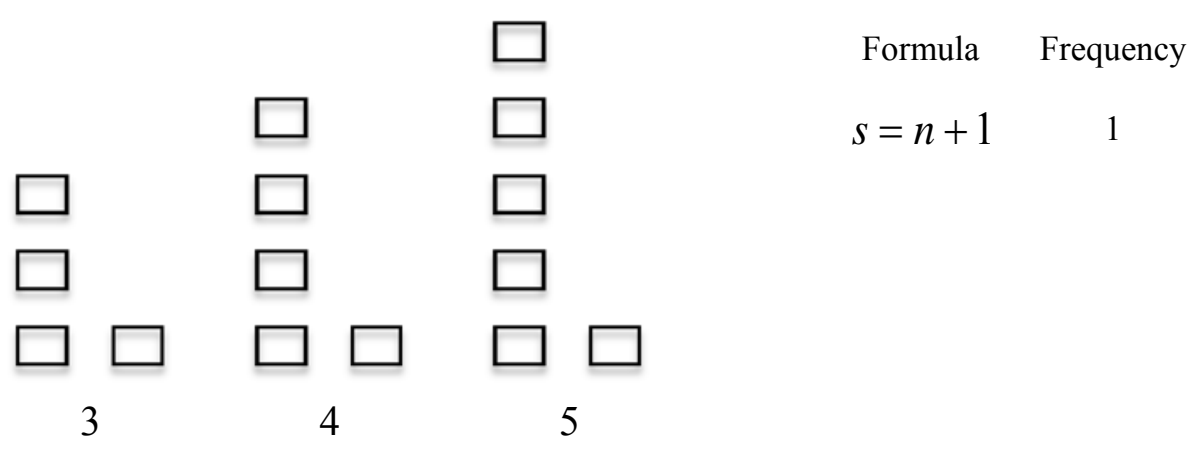

Fig. 16 Students' Patterns on the Fig. 15 Semi-Free Task (Multiple answers were accepted)

\subsubsection{Stage Number-Driven PG Conversion and Processing}

All 11 students initially linked their dimensions with stage number, which actually worked in all cases of their constructed linear patterns. For example, in her response below relative to Pattern A in Fig. 16, Emma linked her two dimensions to stage number. 
This is stage 1 [referring to stage 1]. This is stage 2 [the column of 2 squares]. This is stage 3 [the column of 3 squares] and this is stage 4 [the column of 4 squares]. And then so ahm when I figured that, I try to see what's left. So if it's 1 [the remaining square on the row of stage 2], if you subtract the stage number from 1, you get 1. If you subtract 1 from the stage number [stage 3] you get 2 [the remaining 2 squares on the row of stage 3]. If you subtract 1 from this stage number [stage 4], you get 3 [the remaining 3 squares on the row of stage 4]. In Karen's response below relative to Pattern A in Fig. 16, she abduced two dimensions consisting of a corner square and two non-overlapping row and column sets of squares, where the cardinality of each set is one less than the stage number.

I visualized it in groups. [In the case of $x=n-1+n-1+1$ ], group these [the row and column squares excluding the common corner square each set having cardinality $(n-1)]$ and add 1[the corner square].

Still on pattern A in Fig. 16, Delilah in her response below abduced two different but equivalent dimension-2 structures that were also dependent on stage number.

[First formula] $n=2 x-1$. This is step, stage 3 [referring to the row and column squares in stage 3]. There's like two 3s and this one [corner square] overlaps.... [Second formula] Uhm, I'm not sure this is the simplest form. $n=x^{2}-(x-1)^{2}$. Like if you see a [bigger] square and a [smaller] square here [referring to stage 4, where the bigger square has $4^{2}$ squares and the smaller square has $(4-1)^{2}$ squares].

\subsubsection{Complementary Transformation-Based Abductive Structuring Methods}

While all 11 students employed stage number-driven conversion and processing on all the linear patterns they constructed, some of them found it difficult to apply the same method in cases involving nonlinear patterns. For example, Tamara initially tried to "find a way to relate 
grouping and stage number in some way" in the case of her pattern E in Fig. 16, where every succeeding stage $n$ is the union of the preceding $(n-1)^{\text {th }}$ stage and $(n-1)$ pairs of squares (e.g., stage 2 consists of stage 1 and 1 pair of squares, stage 3 contains stage 2 and 2 pairs of squares, stage 4 consists of stage 3 and 3 pairs of squares). She eventually gave up when she could not process her initial early/recursive AC formula further into either a nonstandard function-based AC or standard function-based CM.

Emma's processing of her Pattern D in Fig. 16 illustrates a successful transition from an early/recursive $\mathrm{AC}$ to a standard function-based $\mathrm{CM}$ that was made possible through joint activity with the interviewer. Emma initially abduced a union of columns of squares that relied on stage number, but she got stuck and could not find a way to process her early/recursive AC further into either a nonstandard function-based AC or standard function-based CM.

I know what to write but I don't know how to put it into a formula. Like so if there's $n$ and then you add all the numbers before it. Like if its 5 , you put $n-1, n-2, n-3$, and $n-4$. But I don't know how to put that in a formula..... [If it's stage 6], it's $n$ plus $n-5$ plus $n-4$ all the way to 1.

With additional prompts from interviewer, she eventually developed a standard function-based $\mathrm{CM}$ formula after engaging in a repeated process of constructing two copies of each stage in her pattern, reconfiguring them to form a rectangle that had dimensions $n$ and $(n+1)$, and then dividing by 2 to signify that she was taking away the extra copy.

Frank's processing of Pattern A in Fig. 16 illustrates how transformation-based abductive structuring action could also be used to explain a standard function-based CM formula. His converted formula was $S(x)=2 x-1$, where $S$ refers to the total number of squares in stage $x$. Frank visually processed each L-shape stage in his pattern as a complete rectangular array of 
$n \times 2$ squares. However, he knew that he needed to take away one square from the complete array in order to match the correct number of squares.

\subsubsection{Discussion}

We note two sufficient conditions for a successful PG conversion and processing involving figural patterns. First, linking dimensions to stage number in multiplicative terms is a systematic way of capturing and organizing abduced partonomic relationships. Repeatedly dividing stages into stage number-driven dimensions also strengthens an abductive claim, which is further verified by inductive action. Emma's articulated thinking relative to Pattern A in Fig. 16 exemplifies how a combination of stage-driven abduction and induction enabled her to achieve meaningful conversion from the visual and verbal to the alphanumeric phase.

Second, while having a multiplicative thinking disposition toward structures is a necessary analytical tool, complementary (figural-based) transformative abductive structuring actions or methods could also "lead to 'seeing' [more] regularities and invariants, which then could be generalized" (Dörfler, 2008, p. 159). Delilah used an auxiliary method by adding $(n-1)$ sets of square arrays, which enabled her to imagine full arrays of squares instead of L-shaped figures of squares. Emma also used an auxiliary method that allowed her to see the usefulness of adding extra copies of the stage numbers in processing and converting to her function-based $\mathrm{CM}$ formula. The two students' auxiliary strategies enabled them to further reconfigure their figural stages in ways that permitted them to see how their inferred dimensions were related to some whole unit. Karen and Frank employed a combination of auxiliary and mereological strategies (cf. Duval, 1999). In PG activity, mereological action involves decomposing or deconstructing stages into parts that are then reorganized, producing a reconstructed figure whose dimensions can be easily abduced and justified. Karen perceived two overlapping equal groups of squares, 
while Frank saw two non-overlapping equal columns of squares. They both added and took away an extra square, which enabled them to convert and process their generalization from the visual to the alphanumeric.

\section{General Discussion and Conclusion}

Middle school students are indeed capable of developing well-defined mathematical structures when PG activity shifts from typical textbook-type tasks to open and free patterning tasks with unregulated abductive assumptions. Such open tasks encourage them to focus on essential rules for PG conversion and processing from the initial figural context to the interpreted and relation-derived nonstandard $\mathrm{AC}$ and standard $\mathrm{CM}$ formulas. Results of the preceding two experiments extrapolate necessary and sufficient conditions for PG construction and justification at the middle school level that students can perform simultaneously with success. Multiplicativedriven abductive and inductive actions together demonstrate in necessary terms the emergence of a tentative intensional generalization. A deductive closure of extensional generalizations also needs to take place in order to justify a valid mathematical structure for an emerging pattern.

However, having a multiplicative thinking disposition toward mathematical structures in PG is not sufficient, especially in situations when early recursive AC formulas are used to describe an intensional generalization. Both stage number-driven mapping and transformationbased abductive actions (e.g., use of auxiliary sets and mereological strategies) provide sufficient analytical conditions that can further support the construction and justification of an intensional generalization that takes either a nonstandard AC or standard CM form. When students are able to successfully coordinate both necessary and sufficient conditions, a complete and algebraically useful PG emerges. 
Smith's (2003) point regarding part-to-shape-to-relations resonates well with our findings. That is, among middle school students in PG contexts, the initial phase of seeing parts and seeing wholes in an emerging pattern phenomenologically evolves to seeing mathematical relations and rules in a structural manner over time with notations emerging as objects that are not merely "abstract and intangible" but "perceivable and manipulable" (Dörfler, 2008, p. 159) based on deductively validated relations. This empirical finding supports Dörfler's point that a truly authentic PG activity is "a strongly conceptual activity [that] does not result in a number but in a general conceptual structure presented with variables" ( $p$ 159).

\section{Implications for Future Research}

Our findings raise two issues that have implications for advancing research in $\mathrm{PG}$ in school mathematical contexts. First, this particular line of inquiry that pursues ways in which students construct and justify structures in the context of open or free generalization tasks is new. However, now after the fact, it is important to note that the manner in which we state task conditions that enable all middle school students to engage in such activity meaningfully should not be taken for granted. For example, simply asking students to create and/or extend patterns freely might yield mostly familiar patterns and, hence, would yield predictable results about their thinking and reasoning on patterns that we already know. In our study, the ways in which our students responded to the semi-free tasks reflected instances of patterns that they had previously met, which affected the the range of paths they followed. While we do not undermine their efforts since their thinking still reflected "deep mathematical understanding," we strongly recommend that all future work need to provide students with more directions in order to make such tasks more interesting, less predictable, and more challenging, rigorous, and supportive of deep thinking and further mathematics. ${ }^{\text {vii }}$ For example, asking students to create pattern 
sequences with 1, 2, 3 or more dimensions can test and enhance their PG ability and encourage them to apply both necessary and sufficient conditions in typical and unfamiliar patterning situations that span linear and nonlinear structures. In other words, task conditions can motivate students to think about partonomic relationships (e.g., early recursive, additive composite, contracted multiplicative, etc.) in different ways. The following comment from Duval (2005) is applicable and worth noting in the case of (figural and numerical) patterning activity: "The way of seeing a geometrical figure depends on the activity for what it is used" (p. 5).

Second, one fundamental issue about PG activity that is worth considering in future research investigations involves how to strategically employ analytical tools that can effectively support dimensional (or figural unit) analysis and the transition from early/recursive and nonstandard AC to standard CM forms in both aspects of PG conversion (i.e., involving two or more registers) and processing (i.e., transformations within the same register). In this study, there was minimal use of transformative-based abductive structuring actions that are, in fact, powerful and useful. Research is needed that will determine whether, and how soon, formal experiences in using such tools can help strengthen students' ability to successfully deal with appropriate gradelevel PG tasks. A related concern involves determining the extent to which they occur naturally in students' PG ability.

\section{References}

Anderman, E., Griesinger, T., \& Westerfield, G. (1998). Motivation and cheating during early adolescence. Journal of Educational Psychology, 90(1), 84-93.

Anderman, E. \& Murdock, T. (2006). Psychology of Academic Cheating. Burlington, MA: Elsevier Academic Press. 
Behrmann, M. \& Ewell, C. (2003). Expertise in tactile pattern recognition. Psychological Science, 14(5), 480-486.

Bilalic, M., Langner, R., Erb, M., \& Grodd, W. (2010). Mechanisms and neural basis of object and pattern recognition: A study with chess experts. Journal of Experimental Psychology: General, 139(4), 728-742.

Cadez, T. \& Kolar, V. (2015). Comparison of types of generalizations and problem-solving schemas used to solve a mathematical problem. Educational Studies in Mathematics, 89, 283-306.

Devlin, K. (2008). It ain't no repeated addition. Retrieved online: https://www.maa.org/external_archive/devlin/devlin_06_08.html.

Dörfler, W. (2008). En route from patterns to algebra: Comments and reflections. ZDM, 40(1), $143-160$.

Duval, R. (2014). Commentary: Linking epistemology and semio-cognitive modeling in visualization. ZDM, 46(1), 159-70.

Duval, R. (2005). Les conditions cognitives de l'apprentissage de la géométrie : développement de la visualisation, différenciation des raisonnements et coordination de leur fonctionnement. Annales de didactique et de sciences cognitives, 10, 5-53. Retrieved online:

https://mathinfo.unistra.fr/fileadmin/upload/IREM/Publications/Annales_didactique/vol_ 10/adsc10-2005_001.pdf.

Duval, R. (1999). Representation, vision, and visualization: Cognitive functions in mathematical thinking. In F. Hitt \& M. Santos (eds.), Proceedings of the 21st North American PME Conference (pp. 3-26). Cuernavaca, Morelos, Mexico: PMENA. 
Jurdak, M. \& El Mouhayar, R. (2014). Trends in the development of student level of reasoning in pattern generalization tasks across grade level. Educational Studies in Mathematics, $85,75-92$.

Jüttner, M., Caelli, T., \& Rentschler, I. (1997) Evidence-based pattern classification: A structural approach to human perceptual learning and generalization. Journal of Mathematical Psychology, 41, 244-259.

Larkin, J., McDermott, J., Simon, D., \& Simon, H. (1980). Expert and novice performance in solving physics problems. Science, 208(2), 1335-1342.

Lim, E., Liu, J., \& Lee, R. (2011). Knowledge seeker - ontology modelling for information search and management: A compendium. New York: Springer.

National Governors Association Center for Best Practices, Council of Chief State School Officers. (2010). Common Core State Standards: Mathematics. Washington DC: Author.

National Research Council (2013). The mathematical sciences in 2025. Washington, DC: The National Academies Press.

Nilsson, P. \& Juter, K. (2011). Flexibility and coordination among acts of visualization and analysis in a pattern generalization activity. Journal of Mathematical Behavior, 30, 194205.

Rivera, F. \& Becker, J. (2011). Formation of pattern generalization involving linear figural patterns among middle school students: Results of a three-year study. In J. Cai \& E. Knuth (eds.), Early algebraization: A global dialogue from multiple perspectives (Advances in mathematics education Vol. 2) (pp. 323-366). New York: Springer.

Rivera, F. \& Becker, J. (2009). Algebraic Reasoning Through Patterns. Mathematics Teaching in the Middle School, 15(4), 212-221. 
Rivera, F. (2016). Abduction and the emergence of necessary mathematical knowledge. In L. Magnani and T. Bertolotti (eds.), Springer handbook of model-based science. New York: Springer.

Rivera, F. (2013). Teaching and learning patterns: Psychological and pedagogical perspectives. New York, NY: Springer.

Rivera, F. (2011). Toward a visually-oriented school mathematics curriculum: Research, theory, practice, and issues (Mathematics Education Library Series 49). New York, NY: Springer.

Rivera, F. (2010). Visual Templates in Pattern Generalization Activity. Educational Studies in Mathematics, 73(3), 297-328.

Smith, L. (2003). Learning to recognize objects. Psychological Science, 14(3), 244-250.

Stavy, R. \& Babai, R. (2008). Complexity of shapes and quantitative reasoning in geometry. Mind, Brain, and Education, 2(4), 170-176.

Tanisli, D. \& Özdas, A. (2009). The strategies of using the generalizing patterns of primary school $5^{\text {th }}$ grade students. Educational Sciences: Theory \& Practice, 9(3), 1485-97.

Walkowiak, T. (2014). Elementary and middle schools students' analyses of pictorial growth patterns. Journal of Mathematical Behavior, 33, 56-71.

Wilkie, K. (in press). Students' use of variables and multiple representations in generalizing functional relationships prior to secondary school. Educational Studies in Mathematics.

Wu, H. H. (2011). Understanding numbers in elementary school mathematics. Washington, DC: Mathematical Association of America. 
${ }^{\text {i }}$ Readers are referred to Rivera (2016) for an extensive discussion of the roles of abduction, induction, deduction,
and deductive closure in the emergence of necessary mathematical knowledge.

ii Partonomy "divides concept as a whole into different parts (e.g., India and China are parts of Asia)," which is distinguished from taxonomy that "divides a concept into species of kinds (e.g., Jazz and Classical are types of Music)” (Lim, Liu, \& Lee, 2011, p. 16).

iii The term standard pertains to a way of expressing and writing an expression in its simplified or reduced form.

iv In all our reported and published studies on patterns that pertained to the two cohorts, we explicitly attended to the social (and sociomathematical) norms that the students carefully observed, especially during teaching experiments. One social norm specifically addressed exercising appropriate disposition and mindset toward tasks that required them to respond honestly and independently. The students were, in fact, fully aware that learning mathematics in their Algebra I class did not focus on extrinsic goals (e.g., obtaining good grades, being competitive) but on proficiency goals and various opportunities to learn better and in ways that aligned with the sociomathematical norms (e.g., using class time to discuss together effective and sophisticated ways of solving problem tasks; Rivera, 2011, 2013; cf. Anderman et al., 1998; Anderman \& Murdock, 2006;). We note that the cover sheet that came with the homework assignment included the following line, "Honesty Code: Do This on Your Own," as a reminder. Also, since we were both involved in data analysis, we were especially sensitive to student responses that were alarmingly similar in both PG aspects of construction and justification.

vThe definition of multiplication involving two whole numbers is consistent with the initial (third-grade) definition that is stipulated in the Common Core State Standards in Mathematics (National Governors Association Center for Best Practices, Council of Chief State School Officers (NGACBP/CCSO, 2010; cf. Wu, 2011). While it seemed to be a very restricted approach to multiplicative thinking, the authors employed linear PG tasks involving discrete objects (i.e., pattern blocks) to help them initially develop their ability to convert and process patterns that they could easily manipulate and transform in alphanumeric terms. Beginning with this restricted approach enabled us to avoid unnecessary distractions, but it also enabled the students to see how the concept and process of multiplication could be interpreted as the result of scaling some quantity in linear patterning activity contexts that see such quantities in terms of figural units that repeat and grow according to stage number (cf. Devlin, 2008). Furthermore, the PG activity with the pattern blocks provided the expert cohort of students with an opportunity to teach the novice cohort about the relevant abstract processes that would generate successful patterning conversion and processing in alphanumeric terms. We note that when the students engaged in linear function modeling activity involving domains that spanned the set of rational numbers, they did not experience difficulty transitioning to multiplicative situations whose factors are either or both rational numbers since they understood and employed the same rules and operations - that is, mathematical structure - that they acquired from their experiences with discrete objects. Readers are referred to Rivera (2013) and Rivera \& Becker (2009) for details of this transition since it is beyond the scope of what we intend to report and discuss in this article.

vi Almost all part-driven linear growth patterns that we analyzed clearly model dimension-2 structures on the basis of consistency in both written and alphanumeric responses. There was one student response, however, that perplexed us. Jake's growing star-like pattern in Fig. 8 could be interpreted as having dimension 1 or 3. Jake saw "three sides, [where] the amount of circles on each side is $n$," which could mean 3 identical dimensions that define the structure of the pattern. But a strict interpretation of Jake's converted formula, $p=n \times 3$, could also convey a whole-stage driven pattern that has dimension 1 by adding a stable triangular group of 3 circles at every stage in the pattern.

vii According to the NGACBP/CCSO (2010), deep mathematical understanding involves the ability to "justify, in a way that is appropriate to one's mathematical maturity, why a particular mathematical statement is true or where a mathematical rule comes from. Mathematical understanding and procedural skill are equally important, and both are assessable using mathematical tasks of sufficient richness" (n.p.). 